\title{
WIPP \\ Subsidence Monument \\ Leveling Survey \\ 2004
}

December 2004
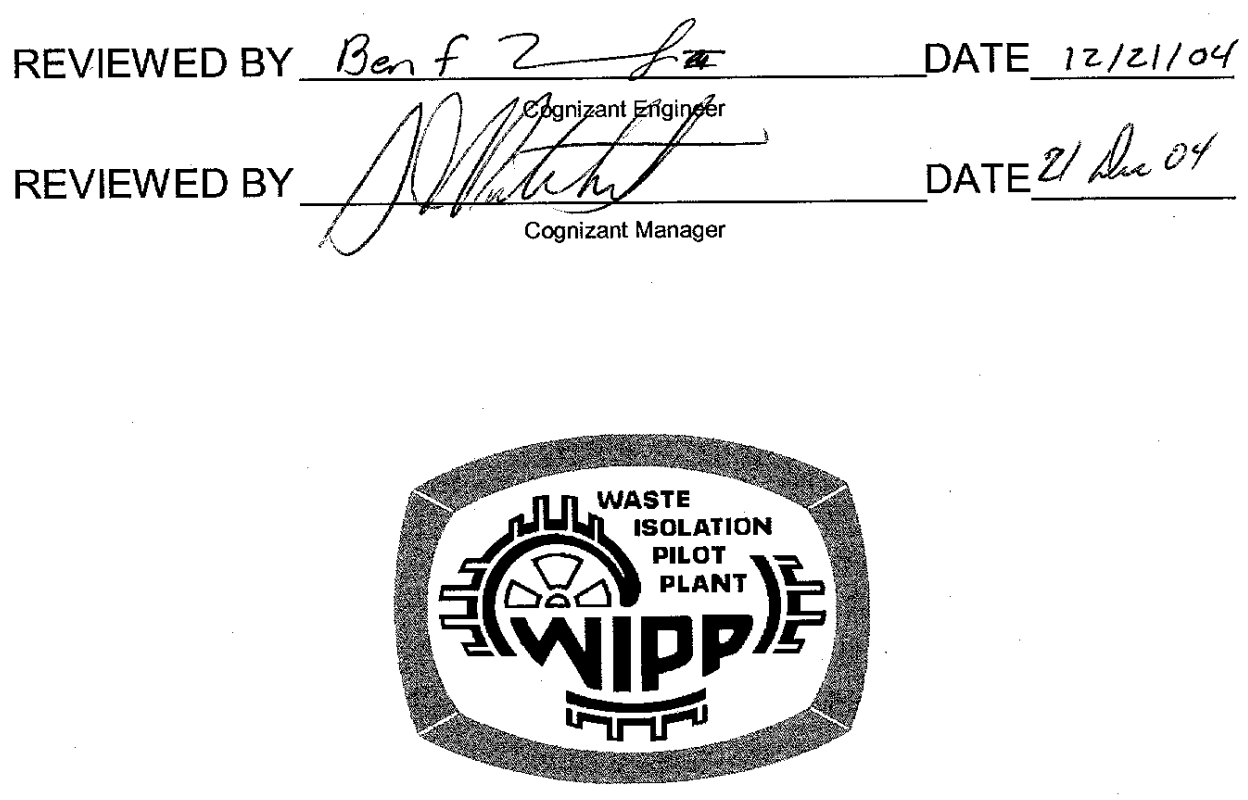

Waste Isolation Pilot Plant 


\section{Table of Contents}

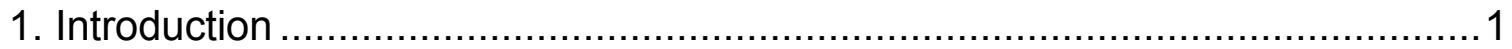

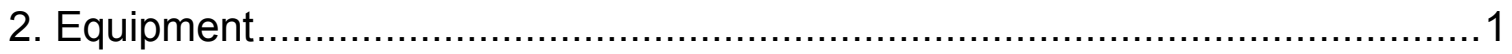

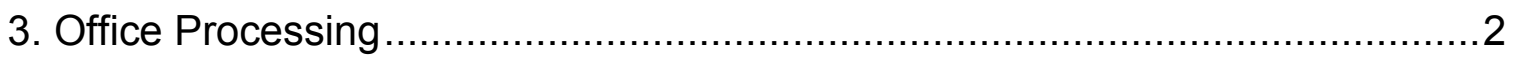

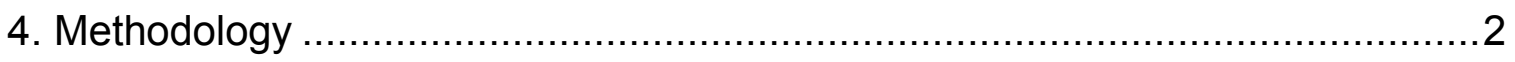

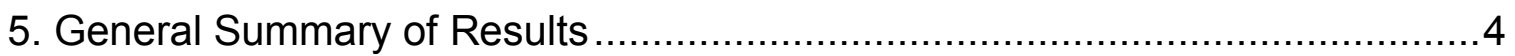

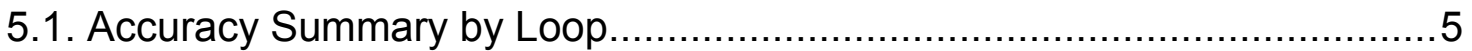

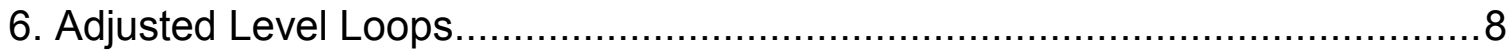

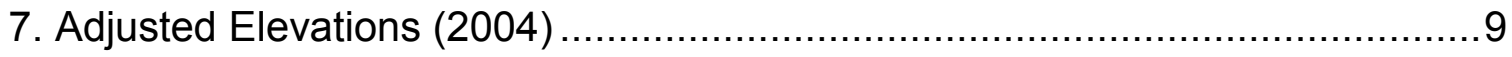

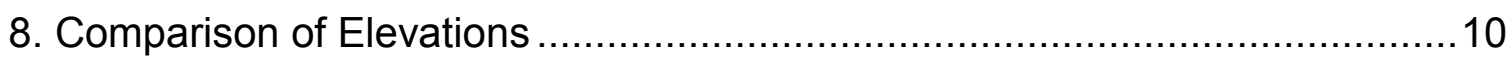

\section{List of Tables}

Table A. Description of 2004 Leveling Loops.............................................. 4

Table B. Summary of Distance and Accuracy for 2004 Leveling Loops............... 4

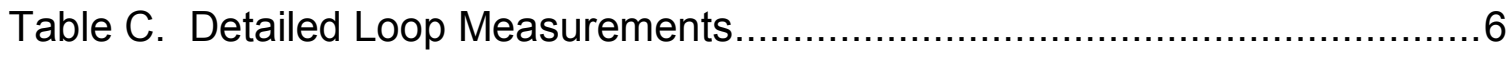

Table D. Adjusted Elevations by Loop ................................................ 8

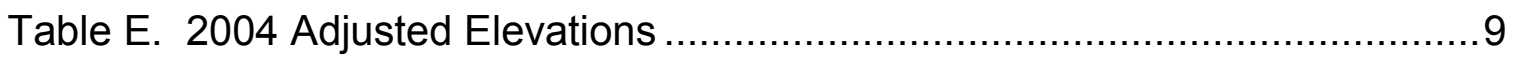

Table F. Comparison of Elevations 1987-2004 ...........................................10

\section{List of Figures}

Figure 1. Individual Loops, Total Loop, and Underground Excavations ............. 3 


\section{List of Acronyms}

DOE Department of Energy

DOY Day of year

FGCS Federal Geodetic Control Subcommittee

M\&TE Measurement and Test Equipment

NGS National Geodetic Survey

WTS Washington TRU Solutions

WIPP Waste Isolation Pilot Plant

\section{References}

Classification, Standards of Accuracy, and General Specifications of Geodetic Control Surveys, Federal Geodetic Control Committee (now Federal Geodetic Control Subcommittee), [1975] 1980, Reprint.

FGCS Specifications and Procedures to Incorporate Electronic Digital / Bar-Code Leveling Systems, Federal Geodetic Control Subcommittee, ver. 4.0, dated July 15,1994 .

WP 09-ES4001, Subsidence Survey Data Acquisition and Report, June 2002

Subsidence Monitoring Software Quality Assurance Plan, July 2002

WIPP Subsidence Monument Leveling Surveys 1986-1997, DOE / WIPP 982293, June 1998.

WIPP Subsidence Monument Leveling Surveys 1998, DOE / WIPP 99-2293, October 1998.

WIPP Subsidence Monument Leveling Surveys 1999, DOE / WIPP 00-2293, October 1999.

WIPP Subsidence Monument Leveling Surveys 2000, DOE / WIPP 01-2293, October 2000

WIPP Subsidence Monument Leveling Surveys 2001, DOE / WIPP 02-2293, October 2001

WIPP Subsidence Monument Leveling Surveys 2002, DOE / WIPP 03-2293, October 2002

WIPP Subsidence Monument Leveling Surveys 2003, DOE / WIPP 04-2293, October 2003 


\section{Introduction}

Sections 2 through 7 of this report define the result of the 2004 leveling survey through the subsidence monuments at the WIPP site. Approximately 15 miles of leveling was completed through nine vertical control loops. The 2004 survey includes the determination of elevation on each of the 48 existing subsidence monuments and the WIPP baseline survey, and 14 of the National Geodetic Survey's (NGS) vertical control points. The field observations were completed during August through November of 2004 by personnel from the Washington TRU Solutions (WTS) Surveying Group, Mine Engineering Department. Additional rod personnel were provided by the Geotechnical Engineering department.

Digital leveling techniques were utilized to achieve better than Second Order Class II loop closures as outlined by the Federal Geodetic Control Subcommittee (FGCS). Because it is important to perform the subsidence survey in exactly the same manner each year, WIPP procedure (WP 09-ES4001) details each step of the survey. Starting with the 2002 survey this procedure has been used to perform the subsidence survey.

Starting with the survey of the year 2001, Loop 1 and redundant survey connections among the various loops were removed from the survey and report. This resulted in a reduction of fieldwork with no loss of accuracy or precision. The redundant connections caused multiple elevations for the same stations. The differences were so slight that they were not used in elevation adjustments for the loops. The redundancy was used to spot gross errors in the field. After several years of surveying these loops it is evident that no gross errors occur that are not also evident in the loop closures.

Finally, Section 8 contains Table F, which summarizes the elevations for all surveys from 1987 through 2004, inclusive. A detailed listing of the 1986 through 1997 surveys is contained in the report, WIPP Subsidence Monument Leveling Surveys 1986-1997, DOE/WIPP 98-2293. A reference to the summary reports for each year after 1997 is listed in the reference section of this document.

\section{Equipment}

The observations were taken with the WILD NA3003 Electronic Digital Level (WIPP M\&TE ID\# DM0999 and DM1002) manufactured by Leica, and bar coded leveling staffs. The calibration date for DM0999 is valid from February 11, 2003, through February 11, 2005. The calibration for the DM1002 is valid from March 22,2004 , through March 22, 2006. The data were recorded electronically on the Leica GRM10 REC-Module, which plugs directly into the instrument. In addition to the electronic record, a written field log was maintained to record information that is not stored in the electronic record. 


\section{Office Processing}

Each day the data were downloaded from the GRM10 REC-Module to the survey group computer. The original raw data files were maintained intact, and further processing was performed on a copy of the original raw data file.

Listing of the data, and the adjustment of the loops, was completed with the DIGILEV software (version 10.94d) from Leica Canada. The results, as summarized below, were extracted from the output of the DIGILEV software. A Software Quality Assurance Plan was written for the computer programs used in reducing the subsidence survey field notes. DIGILEV was tested, verified and validated. The program was deemed acceptable and is now in the WIPP controlled software list.

\section{Methodology}

The weather conditions during the observations of the 2004 survey were generally mild with moderate temperatures and light to moderate breezes.

The elevations for the 2004 survey are computed from the adjusted observations based on the elevation of the subsidence monument, S-37 (3,423.874 feet). $\mathrm{S}-37$ is the WIPP monument that is furthest from the influence of the underground excavations, and has been held fixed for all of the subsidence leveling surveys since 1993. The condition of the individual monuments was substantially the same as the previous subsidence survey with three exceptions.

The brass cap of monument PT-31 came loose making it unusable and monuments S-17 and S-18 no longer exist due to construction of the new salt storage/disposal pad.

As in previous years, the subsidence survey was divided into nine loops. Each loop generally takes one day to complete. This allows a loop to be completed in one surveying session and results in a lower probability of error.

For visual reference, Figure 1 shows a graphic display of the individual loops, the total survey, and the relationship to the underground excavations. 


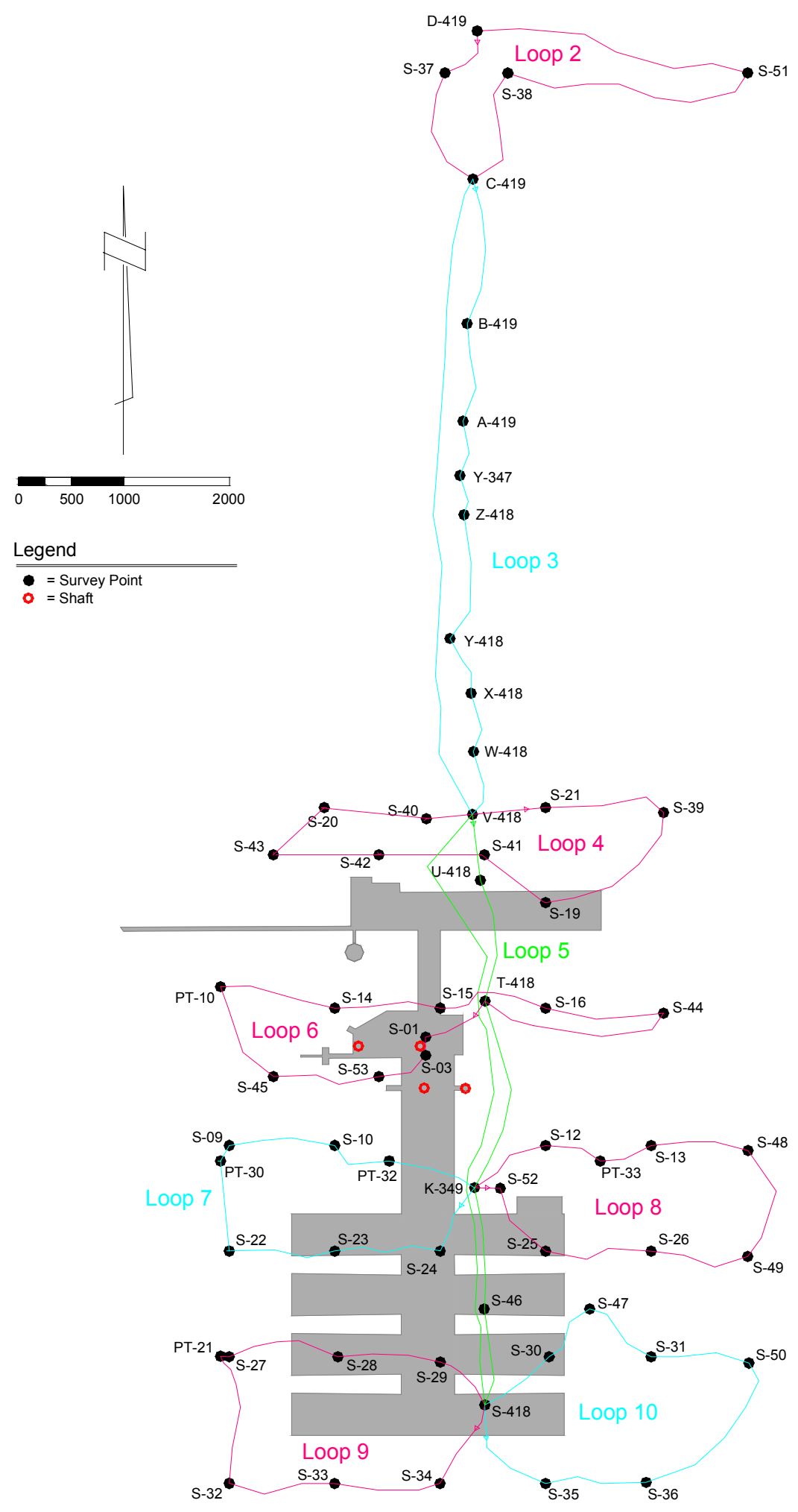

Figure 1. Individual Loops, Total Survey and Underground Excavations 


\section{General Summary of Results}

Table A below describes the nine leveling loops that were measured to obtain the elevations of the subsidence monuments. The table contains the start date of the observations, a loop number, and the points that are contained within the loop.

Table A. Description of 2004 Leveling Loops

\begin{tabular}{|c|c|c|}
\hline $\begin{array}{c}\begin{array}{c}\text { Start Date } \\
\text { (DOY) }\end{array} \\
\end{array}$ & Loop & Points \\
\hline $\begin{array}{c}\text { August 31, } 2004 \\
(243)\end{array}$ & 2 & D-419, S-37, C-419, S-38, S-51, D-419 \\
\hline $\begin{array}{l}\text { September } 17 \& 23,2004 \\
(260 \text { \& 266) }\end{array}$ & 3 & $\begin{array}{l}\text { C-419, B-419, A-419, Y-347, Z-418, Y-418, X-418, } \\
\text { W-418, V-418, C-419 }\end{array}$ \\
\hline $\begin{array}{c}\text { October 1, } 2004 \\
(274)\end{array}$ & 4 & $\begin{array}{l}\text { V-418, S-21, S-39, S-19, S-41, S-42, S-43, S-20, S-40, } \\
\text { V-418 }\end{array}$ \\
\hline $\begin{array}{c}\text { September 27, } 2004 \\
(270)\end{array}$ & 5 & V-418, U418, T-418, K-349, S-46, S-418, V-418 \\
\hline $\begin{array}{c}\text { December 9,2004 } \\
(343)\end{array}$ & 6 & $\begin{array}{l}\text { T-418, S-01, S-03, S-53, S-45, PT-10, S-14, S-15, } \\
\text { S-16, S-44, T-418 }\end{array}$ \\
\hline $\begin{array}{l}\text { October 12, } 2004 \\
(285)\end{array}$ & 7 & $\begin{array}{l}\text { K-349, S-24, S-23, S-22, PT-30, S-09, S-10, PT-32, K- } \\
349\end{array}$ \\
\hline $\begin{array}{l}\text { October 28, } 2004 \\
(301)\end{array}$ & 8 & $\begin{array}{l}\text { K-349, S-52, S-25, S-26, S-49, S-48, S-13, PT-33, } \\
\text { S-12, K-349 }\end{array}$ \\
\hline $\begin{array}{c}\text { December 1, } 2004 \\
(335)\end{array}$ & 9 & $\begin{array}{l}\text { S-418, S-34, S-33, S-32, PT-21, S-27, S-28, S-29, } \\
\text { S-418 }\end{array}$ \\
\hline $\begin{array}{c}\text { November 18, } 2004 \\
(322)\end{array}$ & 10 & S-418, S-35, S-36, S-50, S-31, S-47, S-30, S-418 \\
\hline
\end{tabular}

Table B summarizes the results of the leveling loops in terms of vertical closure and accuracy. The requirement for Second Order Class II loop closure accuracy was achieved in all cases.

Table B. Summary of Distance and Accuracy for 2004 Leveling Loops

\begin{tabular}{|c|c|c|c|c|}
\hline \hline Loop & $\begin{array}{c}\text { Cumulative } \\
\text { Distance (ft.) }\end{array}$ & $\begin{array}{c}\text { Vertical } \\
\text { Closure (ft.) }\end{array}$ & $\begin{array}{c}\text { Accuracy } \\
\text { (ft.Vmile) }\end{array}$ & $\begin{array}{c}\text { Allowable } \\
\text { Accuracy } \\
\text { (ft. } \text { mile) }\end{array}$ \\
\hline \hline 2 & $8,361.46$ & -0.0021 & 0.002 & 0.042 \\
\hline 3 & $12,704.10$ & -0.0009 & 0.001 & 0.051 \\
\hline 4 & $8,448.77$ & 0.0061 & 0.005 & 0.042 \\
\hline 5 & $13,384.83$ & -0.0033 & 0.002 & 0.053 \\
\hline 6 & $10,012.47$ & 0.0042 & 0.003 & 0.045 \\
\hline 7 & $6,699.67$ & 0.0010 & 0.001 & 0.037 \\
\hline 8 & $7,170.20$ & -0.0049 & 0.004 & 0.038 \\
\hline 9 & $7,525.99$ & 0.0001 & 0.000 & 0.039 \\
\hline 10 & $6,974.18$ & 0.0037 & 0.003 & 0.038 \\
\hline
\end{tabular}




\subsection{Accuracy Summary by Loop}

Table $C$ shows a detailed summary of the observations in the leveling loops for the 2004 survey. All results are shown in feet. The information in the table for each loop includes:

Between each benchmark in the loop:

- The distance leveled between benchmarks along the loop.

- The number of instrument setups between each of the benchmarks.

- The difference in elevation from each benchmark to the next.

For each loop as a whole:

- The cumulative, or total, distance of each loop.

- The vertical closure of the loop.

- The accuracy of leveling.

- Allowable accuracy for each loop.

The accuracy of the leveling is given in terms of feet times the square root of the length of the loop in miles. The actual accuracy of leveling is computed in the DIGILEV software, and is based on the actual vertical closure of the loop. The maximum allowable accuracy is based on the allowable accuracy of a loop as stated in the FGCS specification for digital leveling. The FGCS specification for Second Order Class II loop closure permits a maximum of $8 \mathrm{~mm} \sqrt{\mathrm{Km}}(8 \mathrm{~mm}$ times the square root of the length of the loop in $\mathrm{Km}$ ). This converts to $0.033 \mathrm{ft}$. $\sqrt{\mathrm{mile}}$ ( 0.033 feet times the square root of the length of the loop in miles) when stated in English System. All values indicated in this summary are expressed in feet.

Inspection of the following tables shows that in every case the actual accuracy is well below the maximum allowable accuracy for each loop. The column in each table that is labeled "Difference" is the vertical difference from one point to the next. It is important to note that the vertical difference figures have been rounded, and a slight difference may exist in the vertical closure figure from the algebraic sum of the column. 
Table C. Detailed Loop Measurements

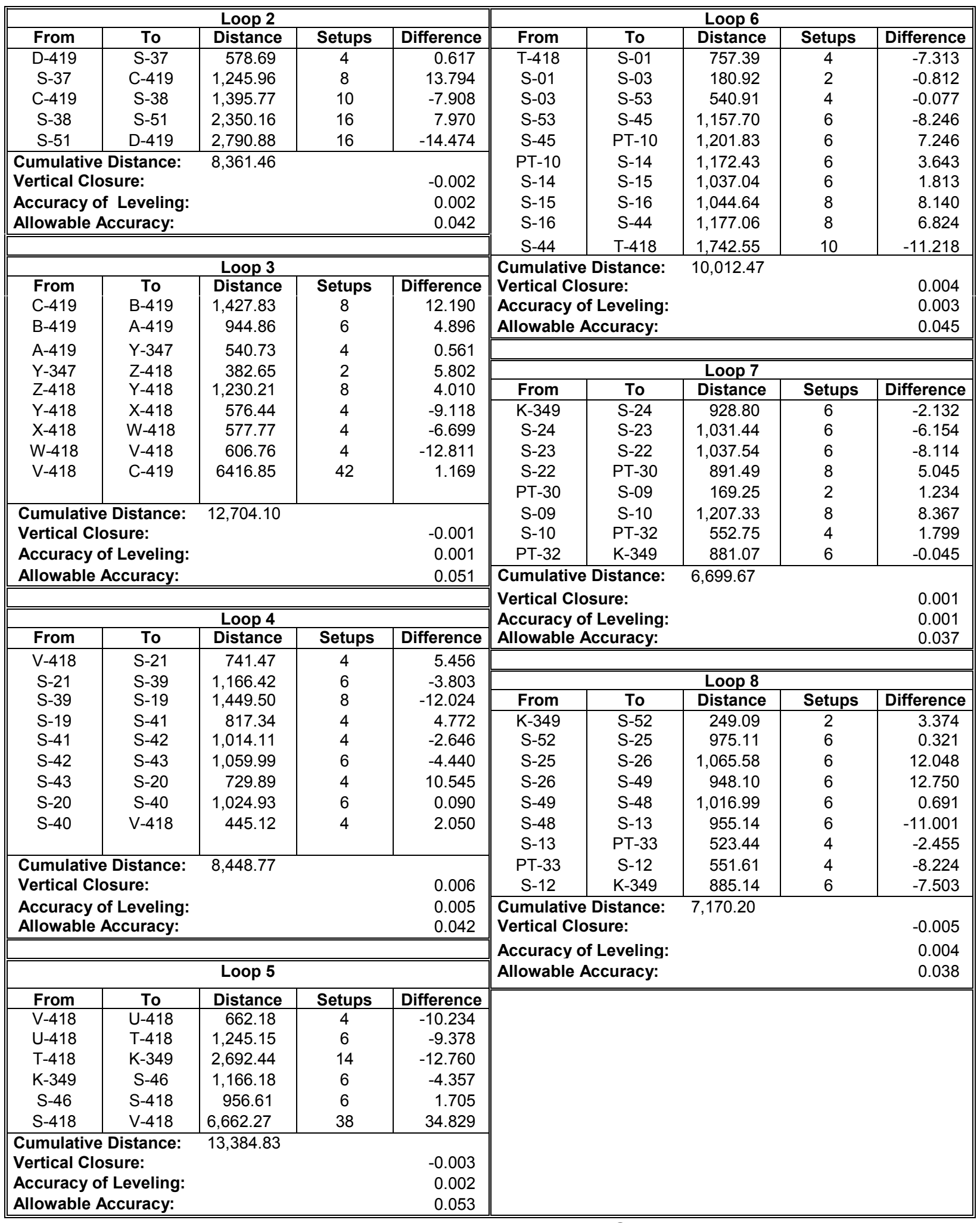

Table C continued on next page... 
DOE/WIPP 05-2293

Table C. Detailed Loop Measurements (continued)

\begin{tabular}{|c|c|c|c|c|c|c|c|c|c|}
\hline \multicolumn{5}{|c|}{ Loop 9} & \multicolumn{5}{|c|}{ Loop 10} \\
\hline From & To & Distance & Setups & Difference & From & To & Distance & Setups & Difference \\
\hline S-418 & S-34 & $1,004.80$ & 6 & -9.615 & S-418 & S-35 & $1,327.72$ & 8 & -1.157 \\
\hline S-34 & S-33 & $1,024.86$ & 6 & -13.035 & S-35 & S-36 & 990.42 & 6 & 9.035 \\
\hline S-33 & S-32 & $1,129.56$ & 6 & -5.581 & S-36 & S-50 & $1,515.11$ & 8 & 16.327 \\
\hline S-32 & PT-21 & $1,290.20$ & 8 & 10.435 & S-50 & S-31 & 954.14 & 6 & -13.622 \\
\hline PT-21 & S-27 & 234.80 & 2 & 3.357 & S-31 & S-47 & 746.11 & 4 & -3.106 \\
\hline S-27 & S-28 & $1,062.86$ & 6 & 6.079 & S-47 & S-30 & 611.43 & 4 & -5.216 \\
\hline S-28 & S-29 & 979.00 & 6 & 6.686 & S-30 & S-418 & 829.25 & 6 & -2.262 \\
\hline S-29 & S-418 & 799.91 & 6 & 1.674 & \multirow{3}{*}{\multicolumn{2}{|c|}{$\begin{array}{l}\text { Cumulative Distance: } \\
\text { Vertical Closure: } \\
\text { Accuracy of Leveling: } \\
\text { Allowable Accuracy: }\end{array}$}} & \multicolumn{2}{|l|}{$6,974.18$} & \\
\hline \multirow{2}{*}{\multicolumn{2}{|c|}{$\begin{array}{l}\text { Cumulative Distance: } \\
\text { Vertical Closure: } \\
\text { Accuracy of Leveling: }\end{array}$}} & \multirow{2}{*}{\multicolumn{2}{|c|}{$7,525.99$}} & 0.000 & & & & & 0.004 \\
\hline & & & & $\begin{array}{l}0.000 \\
0.039\end{array}$ & & & & & 0.038 \\
\hline
\end{tabular}




\section{Adjusted Level Loops}

Table $\mathrm{D}$ is a summary of the adjusted elevations for the nine loops measured in 2004. They have been extracted from the output of the DIGILEV software. These are adjusted elevations within each loop. These final adjusted elevations also appear in Table E

Table D. Adjusted Elevations by Loop

\begin{tabular}{|c|c|c|c|c|c|}
\hline \multicolumn{2}{|c|}{ Loop 2} & \multicolumn{2}{|c|}{ Loop 5} & \multicolumn{2}{|c|}{ Loop 8} \\
\hline D-419 & 3423.257 & V-418 & 3436.499 & $\mathrm{~K}-349$ & 3404.127 \\
\hline S-37 & 3423.874 & $\mathrm{U}-418$ & 3426.265 & S-52 & 3407.501 \\
\hline C-419 & 3437.668 & $\mathrm{~T}-418$ & 3416.887 & S-25 & 3407.822 \\
\hline S-38 & 3429.761 & $\mathrm{~K}-349$ & 3404.127 & S-26 & 3419.870 \\
\hline S-51 & 3437.730 & S-46 & 3399.770 & S-49 & 3432.620 \\
\hline D-419 & 3423.257 & S-418 & 3401.670 & S-48 & 3433.311 \\
\hline & & $\mathrm{V}-418$ & 3436.499 & S-13 & 3422.310 \\
\hline \multicolumn{2}{|c|}{ Loop 3} & & & PT-33 & 3419.855 \\
\hline C-419 & 3437.668 & \multicolumn{2}{|c|}{ Loop 6} & S-12 & 3411.630 \\
\hline B-419 & 3449.858 & $\mathrm{~T}-418$ & 3416.887 & K-349 & 3404.127 \\
\hline A-419 & 3454.754 & S-01 & 3409.575 & \multirow{2}{*}{\multicolumn{2}{|c|}{ Loop 9}} \\
\hline $\mathrm{Y}-347$ & 3455.315 & S-03 & 3408.762 & & \\
\hline Z-418 & 3461.117 & S-53 & 3408.686 & S-418 & 3401.670 \\
\hline$Y-418$ & 3465.126 & S-45 & 3400.440 & S-34 & 3392.055 \\
\hline$X-418$ & 3456.009 & PT-10 & 3407.685 & S-33 & 3379.020 \\
\hline W-418 & 3449.310 & S-14 & 3411.329 & S-32 & 3373.439 \\
\hline V-418 & 3436.499 & S-15 & 3413.142 & PT-21 & 3383.874 \\
\hline \multirow[t]{4}{*}{ C-419 } & 3437.668 & S-16 & 3421.281 & S-27 & 3387.231 \\
\hline & & S-44 & 3428.105 & S-28 & 3393.310 \\
\hline & & T-418 & 3416.887 & S-29 & 3399.996 \\
\hline & & & & S-418 & 3401.670 \\
\hline \multicolumn{2}{|c|}{ Loop 4} & & & & \\
\hline V-418 & 3436.499 & \multicolumn{2}{|c|}{ Loop 7} & \multicolumn{2}{|c|}{ Loop 10} \\
\hline S-21 & 3441.955 & K-349 & 3404.127 & S-418 & 3401.670 \\
\hline S-39 & 3438.152 & S-24 & 3401.995 & S-35 & 3400.514 \\
\hline S-19 & 3426.128 & S-23 & 3395.841 & S-36 & 3409.549 \\
\hline S-41 & 3430.900 & S-22 & 3387.727 & S-50 & 3425.876 \\
\hline S-42 & 3428.254 & PT-30 & 3392.772 & S-31 & 3412.254 \\
\hline S-43 & 3423.814 & S-09 & 3394.006 & S-47 & 3409.149 \\
\hline S-20 & 3434.359 & S-10 & 3402.373 & S-30 & 3403.932 \\
\hline S-40 & 3434.449 & PT-32 & 3404.172 & S-418 & 3401.670 \\
\hline V-418 & 3436.499 & $\mathrm{~K}-349$ & 3404.127 & & \\
\hline & & & & & \\
\hline
\end{tabular}




\section{Adjusted Elevations (2004)}

Table $\mathrm{E}$ shows the adjusted elevations for the subsidence monuments and the NGS points contained within the 2004 survey. These elevations are normalized to the monument, S-37. All elevations are shown in feet, and are within the WIPP local coordinate system.

Table E. 2004 Adjusted Elevations

\begin{tabular}{||c|c||c|c||}
\hline Point & Elevation (ft.) & Point & Elevation (ft.) \\
\hline S-01 & 3409.575 & S-42 & 3428.254 \\
\hline S-03 & 3408.762 & S-43 & 3423.814 \\
\hline S-09 & 3394.006 & S-44 & 3428.105 \\
\hline S-10 & 3402.373 & S-45 & 3400.440 \\
\hline S-12 & 3411.630 & S-46 & 3399.770 \\
\hline S-13 & 3422.310 & S-47 & 3409.149 \\
\hline S-14 & 3411.329 & S-48 & 3433.311 \\
\hline S-16 & 3413.142 & S-49 & 3432.620 \\
\hline S-17 & No longer exists & S-50 & 3425.876 \\
\hline S-18 & No longer exists & S-52 & 3437.730 \\
\hline S-19 & 3426.128 & S-53 & 3407.501 \\
\hline S-20 & 3434.359 & \multicolumn{2}{|c||}{} \\
\hline S-21 & 3441.955 & PT-10 & 3407.685 \\
\hline S-22 & 3387.727 & PT-21 & 3383.874 \\
\hline S-23 & 3395.841 & PT-31 & Monument \\
\hline S-24 & 3401.995 & PT-32 & 3404.172 \\
\hline S-25 & 3407.822 & PT-33 & 3419.855 \\
\hline S-26 & 3419.870 & \multicolumn{2}{|c||}{} \\
\hline S-27 & 3387.231 & S-418 & 3401.670 \\
\hline S-28 & 3393.310 & T-418 & 3416.887 \\
\hline S-29 & 3399.996 & U-418 & 3426.265 \\
\hline S-30 & 3403.932 & V-418 & 3436.499 \\
\hline S-31 & 3412.254 & W-418 & 3449.310 \\
\hline S-32 & 3373.439 & X-418 & 3456.009 \\
\hline S-33 & 3379.020 & Y-347 & 3455.315 \\
\hline S-34 & 3392.055 & Y-418 & 3465.126 \\
\hline S-35 & 3400.514 & Z-418 & 3461.117 \\
\hline S-37 & 3409.549 & A-419 & 3454.754 \\
\hline S-38 & 3423.874 & B-419 & 3449.858 \\
\hline S-40 & 3429.761 & C-419 & 3437.668 \\
\hline S-41 & 3438.152 & D-419 & 3423.257 \\
\hline & 3430.900 & K-349 & 3404.127 \\
\hline & \multicolumn{3}{|c||}{} \\
\hline
\end{tabular}




\section{Comparison of Elevations*}

Table $\mathrm{F}$ compares the elevations from all of the subsidence leveling surveys from 1987 through 2004. All elevations are shown in feet.

Table F. Comparison of Elevations 1987-2004

\begin{tabular}{||c|c|c|c|c|c|c|c|c|c||}
\hline & S-01 & S-02 & S-03 & S-09 & S-10 & S-11 & S-12 & S-13 & S-14 \\
\hline $\mathbf{1 9 8 7}$ & $3,409.738$ & $3,408.219$ & $3,408.914$ & $3,394.056$ & $3,402.466$ & $3,406.437$ & $3,411.790$ & $3,422.428$ & $3,411.500$ \\
\hline $\mathbf{1 9 8 9}$ & $3,409.719$ & $3,411.907$ & $3,408.900$ & $3,394.046$ & $3,402.459$ & $3,406.408$ & $3,411.739$ & $3,422.413$ & $3,411.483$ \\
\hline $\mathbf{1 9 9 2}$ & $3,409.695$ & $3,411.904$ & $3,408.875$ & $3,394.053$ & $3,402.440$ & $3,406.372$ & $3,411.727$ & $3,422.412$ & $3,411.439$ \\
\hline $\mathbf{1 9 9 3}$ & $3,409.616$ & $(1)(2)$ & $3,408.797$ & $3,393.969$ & $3,402.365$ & $(3)$ & $3,411.630$ & $3,422.324$ & $3,411.382$ \\
\hline $\mathbf{1 9 9 4}$ & $3,409.626$ & & $3,408.806$ & $3,393.988$ & $3,402.374$ & & $3,411.653$ & $3,422.348$ & $3,411.372$ \\
\hline $\mathbf{1 9 9 5}$ & $3,409.613$ & & $3,408.795$ & $3,393.986$ & $3,402.373$ & & $3,411.650$ & $3,422.345$ & $3,411.376$ \\
\hline $\mathbf{1 9 9 6}$ & $3,409.615$ & & $3,408.795$ & $3,393.994$ & $3,402.373$ & & $3,411.645$ & $3,422.340$ & $3,411.369$ \\
\hline $\mathbf{1 9 9 7}$ & $3,409.610$ & & $3,408.793$ & $3,394.002$ & $3,402.379$ & & $3,411.656$ & $3,422.349$ & $3,411.368$ \\
\hline $\mathbf{1 9 9 8}$ & $3,409.617$ & & $3,408.802$ & $3,394.011$ & $3,402.388$ & & $3,411.653$ & $3,422.352$ & $3,411.374$ \\
\hline $\mathbf{1 9 9 9}$ & $3,409.613$ & & $3,408.798$ & $3,394.004$ & $3,402.385$ & & $3,411.650$ & $3,422.358$ & $3,411.365$ \\
\hline $\mathbf{2 0 0 0}$ & $3,409.607$ & & $3,408.792$ & $3,394.003$ & $3,402.381$ & & $3,411.644$ & $3,422.352$ & $3,411.364$ \\
\hline $\mathbf{2 0 0 1}$ & $3,409.599$ & & $3,408.786$ & $3,394.006$ & 3402.378 & & $3,411.636$ & $3,422.350$ & $3,411.361$ \\
\hline $\mathbf{2 0 0 2}$ & $3,409.595$ & & $3,408.783$ & $3,394.012$ & $3,402.381$ & & $3,411.637$ & $3,422.354$ & $3,411.357$ \\
\hline $\mathbf{2 0 0 3}$ & $3,409.583$ & & $3,408.771$ & $3,394.007$ & $3,402.372$ & & $3,411.629$ & $3,422.307$ & $3,411.351$ \\
\hline $\mathbf{2 0 0 4}$ & 3.409 .575 & & 3.408 .762 & 3.394 .006 & 3.402 .373 & & 3.411 .630 & 3.422 .310 & 3.411 .329 \\
\hline
\end{tabular}

Note: (1) The subsidence monument, S-02 was relocated in 1989.

(2) The subsidence monument, S-02, no longer exists after the 1992 survey.

(3) The subsidence monument, S-11, no longer exists after the 1992 survey.

\begin{tabular}{|c|c|c|c|c|c|c|c|c|c|}
\hline & S-15 & S-16 & S-17 & S-18 & S-19 & S-20 & S-21 & S-22 & S-23 \\
\hline 1987 & ,413.291 & 421.378 & $3,422.519$ & $3,425.010$ & $3,426.235$ & $3,434.464$ & $3,442.030$ & $3,387.786$ & $3,395.914$ \\
\hline 1989 & & & & & & & & $3,387.795$ & \\
\hline 1992 & & 3,42 & 3,42 & 64 & & & & $3,387.788$ & .028 \\
\hline 1993 & 413.185 & $3,421.256$ & $3,422.404$ & $3,424.859$ & $3,426.136$ & 3,434 & 3,44 & $3,387.701$ & $3,395.853$ \\
\hline 1994 & 413.188 & $3,421.261$ & $3,422.402$ & $3,424.852$ & $3,426.134$ & $3,434.339$ & $3,441.932$ & $3,387.732$ & $3,395.886$ \\
\hline 1995 & 3,413 & 3,421 . & $3,422.4$ & 3,424 & 3,426 & 3,43 & 3,44 & $3,387.727$ & $3,395.877$ \\
\hline 1996 & 3,413 & 3,421 . & $3,422.4$ & 3,42 & 3,4 & 3,43 & $\overline{3,44}$ & $3,387.727$ & $3,395.885$ \\
\hline 1997 & 3,41 & 3,42 & $3,422.431$ & $3,424.864$ & 3,426 & $3,434.346$ & 3,44 & $3,387.738$ & $3,395.889$ \\
\hline 1998 & 34 & 71 & 3,42 & 3,42 & 3,4 & 3,43 & 3,4 & $3,387.744$ & $3,395.887$ \\
\hline 1999 & 77 & 3,4 & 3,422 & 3,42 & 3,42 & $\overline{3,43}$ & 3,4 & $3,387.729$ & $3,395.873$ \\
\hline 2000 & 72 & $\overline{3,42}$ & 3,42 & 3,42 & 3,42 & 3,43 & 3,4 & $3,387.727$ & $3,395.861$ \\
\hline 2001 & & & 3,422 & 3,42 & & & & $3,387.728$ & 857 \\
\hline 2002 & & & $3,422.434$ & 3,424 & & & & $3,387.731$ & $3,395.857$ \\
\hline 2003 & 3,413 & 3,421 . & $3,422.430$ & $3,424.849$ & 3,426 & 3,43 & 3,44 & $3,387.728$ & $3,395.850$ \\
\hline 2004 & $3,413.142$ & $3,421.281$ & $(4)$ & $(5)$ & $3,426.128$ & $3,434.359$ & $3,441.955$ & $3,387.727$ & $3,395.841$ \\
\hline
\end{tabular}

Note: (4) The subsidence monument, S-17, no longer exists after the 2003 survey.

(5) The subsidence monument, S-18, no longer exists after the 2003 survey.

\begin{tabular}{||c|c|c|c|c|c|c|c|c|c||}
\hline & S-24 & S-25 & S-26 & S-27 & S-28 & S-29 & S-30 & S-31 & S-32 \\
\hline $\mathbf{1 9 8 7}$ & $3,402.201$ & $3,408.036$ & $3,420.010$ & $3,387.280$ & $3,393.414$ & $3,400.111$ & $3,404.082$ & $3,412.315$ & $3,373.513$ \\
\hline $\mathbf{1 9 8 9}$ & $3,402.167$ & $3,408.005$ & $3,419.978$ & $3,387.287$ & $3,393.400$ & $3,400.098$ & $3,404.064$ & $3,412.302$ & $3,373.498$ \\
\hline $\mathbf{1 9 9 2}$ & $3,402.159$ & $3,407.974$ & $3,419.948$ & $3,387.310$ & $3,393.421$ & $3,400.113$ & $3,404.073$ & $3,412.303$ & $3,373.533$ \\
\hline $\mathbf{1 9 9 3}$ & $3,402.042$ & $3,407.870$ & $3,419.854$ & $3,387.181$ & $3,393.287$ & $3,400.008$ & $3,403.958$ & $3,412.206$ & $3,373.396$ \\
\hline $\mathbf{1 9 9 4}$ & $3,402.072$ & $3,407.907$ & $3,419.883$ & $3,387.225$ & $3,393.312$ & $3,400.038$ & $3,403.984$ & $3,412.234$ & $3,373.427$ \\
\hline $\mathbf{1 9 9 5}$ & $3,402.062$ & $3,407.895$ & $3,419.871$ & $3,387.216$ & $3,393.309$ & $3,400.031$ & $3,403.978$ & $3,412.230$ & $3,373.425$ \\
\hline $\mathbf{1 9 9 6}$ & $3,402.074$ & $3,407.897$ & $3,419.875$ & $3,387.213$ & $3,393.316$ & $3,400.037$ & $3,403.979$ & $3,412.221$ & $3,373.411$ \\
\hline $\mathbf{1 9 9 7}$ & $3,402.077$ & $3,407.897$ & $3,419.883$ & $3,387.229$ & $3,393.330$ & $3,400.050$ & $3,403.994$ & $3,412.248$ & $3,373.438$ \\
\hline $\mathbf{1 9 9 8}$ & $3,402.076$ & $3,407.902$ & $3,419.883$ & $3,387.248$ & $3,393.338$ & $3,400.059$ & $3,403.998$ & $3,412.248$ & $3,373.452$ \\
\hline $\mathbf{1 9 9 9}$ & $3,402.067$ & $3,407.898$ & $3,419.886$ & $3,387.229$ & $3,393.322$ & $3,400.053$ & $3,403.990$ & $3,412.252$ & $3,373.429$ \\
\hline $\mathbf{2 0 0 0}$ & $3,402.051$ & $3,407.876$ & $3,419.871$ & $3,387.226$ & $3,393.316$ & $3,400.045$ & $3,403.980$ & $3,412.252$ & $3,373.428$ \\
\hline $\mathbf{2 0 0 1}$ & $3,402.035$ & $3,407.862$ & $3,419.872$ & $3,387.231$ & $3,393.318$ & $3,400.040$ & $3,403.972$ & $3,412.255$ & $3,373.431$ \\
\hline $\mathbf{2 0 0 2}$ & $3,402.029$ & $3,407.858$ & $3,419.877$ & $3,387.231$ & $3,393.316$ & $3,400.034$ & $3,403.968$ & $3,412.258$ & $3,373.433$ \\
\hline $\mathbf{2 0 0 3}$ & $3,402.012$ & $3,407.840$ & $3,419.871$ & $3,387.233$ & $3,393.311$ & $3,400.016$ & $3,403.951$ & $3,412.252$ & $3,373.433$ \\
\hline $\mathbf{2 0 0 4}$ & $3,401.995$ & $3,407.822$ & $3,419.870$ & $3,387.231$ & $3,393.310$ & $3,399.996$ & $3,403.932$ & $3,412.254$ & $3,373.439$ \\
\hline
\end{tabular}

Table $F$ continued on next page... 
DOE/WIPP 05-2293

Table F. Comparison of Elevations 1987-2004 (continued)

\begin{tabular}{|c|c|c|c|c|c|c|c|c|c||}
\hline & S-33 & S-34 & S-35 & S-36 & S-37 & S-38 & S-39 & S-40 & S-41 \\
\hline $\mathbf{1 9 8 7}$ & $3,379.093$ & $3,392.128$ & $3,400.597$ & $3,409.583$ & & & & & \\
\hline $\mathbf{1 9 8 9}$ & $3,379.073$ & $3,392.137$ & $3,400.583$ & $3,409.584$ & $3,423.888$ & $3,429.736$ & & & \\
\hline $\mathbf{1 9 9 2}$ & $3,379.090$ & $3,392.138$ & $3,400.591$ & $3,409.605$ & $3,423.874$ & & $3,438.146$ & $3,434.469$ & $3,430.931$ \\
\hline $\mathbf{1 9 9 3}$ & $3,378.975$ & $3,392.026$ & $3,400.478$ & $3,409.504$ & $3,423.874$ & $3,429.736$ & $3,438.110$ & $3,434.430$ & $3,430.888$ \\
\hline $\mathbf{1 9 9 4}$ & $3,379.006$ & $3,392.042$ & $3,400.490$ & $3,409.518$ & $3,423.874$ & $3,429.740$ & $3,438.115$ & $3,434.425$ & $3,430.888$ \\
\hline $\mathbf{1 9 9 5}$ & $3,379.009$ & $3,392.042$ & $3,400.495$ & $3,409.520$ & $3,423.874$ & $3,429.739$ & $3,438.124$ & $3,434.437$ & $3,430.899$ \\
\hline $\mathbf{1 9 9 6}$ & $3,378.992$ & $3,392.028$ & $3,400.483$ & $3,409.501$ & $3,423.874$ & $3,429.744$ & $3,438.118$ & $3,434.436$ & $3,430.891$ \\
\hline $\mathbf{1 9 9 7}$ & $3,379.019$ & $3,392.057$ & $3,400.516$ & $3,409.533$ & $3,423.874$ & $3,429.745$ & $3,438.127$ & $3,434.444$ & $3,430.894$ \\
\hline $\mathbf{1 9 9 8}$ & $3,379.028$ & $3,392.066$ & $3,400.516$ & $3,409.539$ & $3,423.874$ & $3,429.750$ & $3,438.134$ & $3,434.442$ & $3,430.901$ \\
\hline $\mathbf{1 9 9 9}$ & $3,379.011$ & $3,392.056$ & $3,400.507$ & $3,409.539$ & $3,423.874$ & $3,429.751$ & $3,438.149$ & $3,434.445$ & $3,430.900$ \\
\hline $\mathbf{2 0 0 0}$ & $3,379.012$ & $3,392.053$ & $3,400.505$ & $3,409.541$ & $3,423.874$ & $3,429.754$ & $3,438.145$ & $3,434.445$ & $3,430.902$ \\
\hline $\mathbf{2 0 0 1}$ & $3,379.014$ & $3,392.057$ & $3,400.509$ & $3,409.546$ & $3,423.874$ & $3,429.756$ & $3,438.145$ & $3,434.436$ & $3,430.898$ \\
\hline $\mathbf{2 0 0 2}$ & $3,379.017$ & $3,392.060$ & $3,400.513$ & $3,409.550$ & $3,423.874$ & $3,429.757$ & $3,438.142$ & $3,434.437$ & $3,430.897$ \\
\hline $\mathbf{2 0 0 3}$ & $3,379.016$ & $3,392.057$ & $3,400.511$ & $3,409.546$ & $3,423.874$ & $3,429.760$ & $3,438.130$ & $3,434.425$ & $3,430.892$ \\
\hline $\mathbf{2 0 0 4}$ & $3,379.020$ & $3,392.055$ & $3,400.514$ & $3,409.549$ & $3,423.874$ & 3429.761 & $3,428.152$ & $3,434.449$ & $3,430.900$ \\
\hline
\end{tabular}

\begin{tabular}{||c|c|c|c|c|c|c|c|c|c||}
\hline \hline & S-42 & S-43 & S-44 & S-45 & S-46 & S-47 & S-48 & S-49 & S-50 \\
\hline $\mathbf{1 9 8 7}$ & & & & & & & & & \\
\hline $\mathbf{1 9 8 9}$ & & & & & & & & & \\
\hline $\mathbf{1 9 9 2}$ & $3,428.279$ & $3,423.849$ & $3,428.146$ & $3,400.501$ & $3,399.946$ & $3,409.236$ & $3,433.308$ & $3,432.635$ & $3,425.868$ \\
\hline $\mathbf{1 9 9 3}$ & $3,428.230$ & $3,423.813$ & $3,428.070$ & $3,400.406$ & $3,399.837$ & $3,409.133$ & $3,433.238$ & $3,432.572$ & $3,425.809$ \\
\hline $\mathbf{1 9 9 4}$ & $3,428.228$ & $3,423.820$ & $3,428.066$ & $3,400.419$ & $3,399.865$ & $3,409.163$ & $3,433.264$ & $3,432.596$ & $3,425.830$ \\
\hline $\mathbf{1 9 9 5}$ & $3,428.238$ & $3,423.826$ & $3,428.071$ & $3,400.424$ & $3,399.856$ & $3,409.158$ & $3,433.258$ & $3,432.588$ & $3,425.830$ \\
\hline $\mathbf{1 9 9 6}$ & $3,428.238$ & $3,423.823$ & $3,428.078$ & $3,400.423$ & $3,399.856$ & $3,409.157$ & $3,433.256$ & $3,432.585$ & $3,425.816$ \\
\hline $\mathbf{1 9 9 7}$ & $3,428.249$ & $3,423.815$ & $3,428.084$ & $3,400.428$ & $3,399.877$ & $3,409.181$ & $3,433.274$ & $3,432.600$ & $3,425.846$ \\
\hline $\mathbf{1 9 9 8}$ & $3,428.252$ & $3,423.822$ & $3,428.086$ & $3,400.440$ & $3,399.876$ & $3,409.178$ & $3,433.276$ & $3,432.598$ & $3,425.838$ \\
\hline $\mathbf{1 9 9 9}$ & $3,428.255$ & $3,423.825$ & $3,428.091$ & $3,400.435$ & $3,399.866$ & $3,409.176$ & $3,433.289$ & $3,432.611$ & $3,425.851$ \\
\hline $\mathbf{2 0 0 0}$ & $3,428.254$ & $3,423.820$ & $3,428.095$ & $3,400.434$ & $3,399.842$ & $3,409.168$ & $3,433.288$ & $3,432.606$ & $3,425.854$ \\
\hline $\mathbf{2 0 0 1}$ & $3,428.247$ & $3,423.818$ & $3,428.094$ & $3,400.433$ & $3,399.824$ & $3,409.163$ & $3,433.290$ & $3,432.606$ & $3,425.858$ \\
\hline $\mathbf{2 0 0 2}$ & $3,428.246$ & $3,423.815$ & $3,428.097$ & $3,400.435$ & $3,399.818$ & $3,409.160$ & $3,433.297$ & $3,432.613$ & $3,425.863$ \\
\hline $\mathbf{2 0 0 3}$ & $3,428.236$ & $3,423.805$ & $3,428.090$ & $3,400.430$ & $3,399.790$ & $3,409.147$ & $3,433.294$ & $3,432.610$ & $3,425.857$ \\
\hline $\mathbf{2 0 0 4}$ & $3,428.254$ & $3,423.814$ & $3,428.105$ & $3,400.440$ & $3,399.770$ & $3,409.149$ & $3,433.311$ & $3,432.620$ & $3,425.876$ \\
\hline
\end{tabular}

\begin{tabular}{||c|c|c|c|c|c|c|c|c|c||}
\hline \hline & S-51 & S-52 & S-53 & S-54 & PT-10 & PT-21 & PT-30 & PT-31 & PT-32 \\
\hline $\mathbf{1 9 8 7}$ & & & & & & & & & \\
\hline $\mathbf{1 9 8 9}$ & & & & & & & & & \\
\hline $\mathbf{1 9 9 2}$ & $3,437.765$ & $3,407.611$ & $3,408.775$ & $3,411.085$ & $3,407.722$ & & $3,392.914$ & $3,385.117$ & $3,404.370$ \\
\hline $\mathbf{1 9 9 3}$ & $3,437.746$ & $3,407.523$ & $3,408.670$ & $(6)$ & $3,407.664$ & $3,383.821$ & $3,392.823$ & $3,385.027$ & $3,404.296$ \\
\hline $\mathbf{1 9 9 4}$ & $3,437.749$ & $3,407.542$ & $3,408.709$ & & $3,407.672$ & $3,383.868$ & $3,392.843$ & $3,385.051$ & $3,404.311$ \\
\hline $\mathbf{1 9 9 5}$ & $3,437.746$ & $3,407.542$ & $3,408.702$ & & $3,407.671$ & $3,383.862$ & $3,392.844$ & $3,385.050$ & $3,404.322$ \\
\hline $\mathbf{1 9 9 6}$ & $3,437.729$ & $3,407.536$ & $3,408.704$ & & $3,407.669$ & $3,383.858$ & $3,392.852$ & $3,385.053$ & $3,404.312$ \\
\hline $\mathbf{1 9 9 7}$ & $3,437.725$ & $3,407.544$ & $3,408.702$ & & $3,407.675$ & $3,383.874$ & $3,392.857$ & $3,385.063$ & $3,404.321$ \\
\hline $\mathbf{1 9 9 8}$ & $3,437.724$ & $3,407.549$ & $3,408.714$ & & $3,407.687$ & $3,383.887$ & $(7)$ & $3,385.067$ & $3,404.322$ \\
\hline $\mathbf{1 9 9 9}$ & $3,437.729$ & $3,407.544$ & $3,408.709$ & & $3,407.689$ & $3,383.868$ & & $3,385.053$ & $3,404.315$ \\
\hline $\mathbf{2 0 0 0}$ & $3,437.729$ & $3,407.531$ & $3,408.704$ & & $3,407.685$ & $3,383.868$ & & $3,385.053$ & $3,404.306$ \\
\hline $\mathbf{2 0 0 1}$ & $3,437.731$ & $3,407.522$ & $3,408.701$ & & $3,407.687$ & $3,383.874$ & & $3,385.053$ & $3,404.259$ \\
\hline $\mathbf{2 0 0 2}$ & $3,437.733$ & $3,407.521$ & $3,408.700$ & & $3,407.688$ & $3,383.871$ & & $3,385.057$ & $3,404.250$ \\
\hline $\mathbf{2 0 0 3}$ & $3,437.731$ & $3,407.507$ & $3,408.690$ & & $3,407.685$ & $3,383.874$ & & $3,385.054$ & $3,404.234$ \\
\hline $\mathbf{2 0 0 4}$ & $3,437.730$ & $3,407.501$ & $3,408.686$ & & $3,407.685$ & $3,383.874$ & & $(8)$ & $3,404.172$ \\
\hline
\end{tabular}

Note: (6) The subsidence monument, S-54, no longer exists after the 1992 survey.

(7) The monument, PT-30, has been physically disturbed and was removed from the 1998 survey.

(8) The monument, PT-31, has been physically disturbed and was removed from the 2004 survey.

Table F continued on next page... 
DOE/WIPP 05-2293

Table F. Comparison of Elevations 1987-2004 (continued)

\begin{tabular}{||c|c|c|c|c|c|c|c|c|c||}
\hline & PT-33 & S-418 & T-418 & U-418 & V-418 & W-418 & X-418 & Y-347 & Y-418 \\
\hline $\mathbf{1 9 8 7}$ & & & & & & & & & \\
\hline $\mathbf{1 9 8 9}$ & & & & & & & & & \\
\hline $\mathbf{1 9 9 2}$ & $3,419.939$ & & & & & & & & \\
\hline $\mathbf{1 9 9 3}$ & $3,419.853$ & & & & & & & & \\
\hline $\mathbf{1 9 9 4}$ & $3,419.884$ & & & & & & & & \\
\hline $\mathbf{1 9 9 5}$ & $3,419.869$ & & & & & & & & \\
\hline $\mathbf{1 9 9 6}$ & $3,419.865$ & $3,401.696$ & $3,416.902$ & $3,426.267$ & $3,436.481$ & $3,449.276$ & $3,455.969$ & $3,455.274$ & $3,465.080$ \\
\hline $\mathbf{1 9 9 7}$ & $3,419.873$ & $3,401.708$ & $3,416.906$ & $3,426.272$ & $3,436.487$ & $3,449.282$ & $3,455.976$ & $3,455.281$ & $3,465.091$ \\
\hline $\mathbf{1 9 9 8}$ & $3,419.879$ & $3,401.715$ & $3,416.915$ & $3,426.279$ & $3,436.497$ & $3,449.292$ & $3,455.987$ & $3,455.291$ & $3,465.101$ \\
\hline $\mathbf{1 9 9 9}$ & $3,419.880$ & $3,401.707$ & $3,416.913$ & $3,426.275$ & $3,436.500$ & $3,449.304$ & $3,456.000$ & $3,455.304$ & $3,465.117$ \\
\hline $\mathbf{2 0 0 0}$ & $3,419.872$ & $3,401.702$ & $3,416.911$ & $3,426.273$ & $3,436.502$ & $3,449.307$ & $3,456.005$ & $3,455.309$ & $3,465.123$ \\
\hline $\mathbf{2 0 0 1}$ & $3,419.866$ & $3,401.702$ & $3,416.905$ & $3,426.270$ & $3,436.502$ & $3,449.310$ & $3,456.007$ & $3,455.312$ & $3,465.125$ \\
\hline $\mathbf{2 0 0 2}$ & $3,419.868$ & $3,401.701$ & $3,416.901$ & $3,426.269$ & $3,436.502$ & $3,449.311$ & $3,456.009$ & $3,455.314$ & $3,465.126$ \\
\hline $\mathbf{2 0 0 3}$ & $3,419.866$ & $3,401.685$ & $3,416.892$ & $3,426.264$ & $3,436.500$ & $3,449.308$ & $3,456.007$ & $3,455.312$ & $3,465.125$ \\
\hline $\mathbf{2 0 0 4}$ & $3,419.855$ & $3,401.670$ & $3,416.887$ & $3,426.265$ & $3,436.499$ & $3,449.310$ & $3,456.009$ & $3,455.315$ & $3,465.126$ \\
\hline
\end{tabular}

\begin{tabular}{|l|c|c|c|c|c|c|c|c|c||}
\hline & Z-418 & A-419 & B-419 & C-419 & D-419 & K-349 & & & \\
\hline $\mathbf{1 9 8 7}$ & & & & & & & & & \\
\hline $\mathbf{1 9 8 9}$ & & & & & & & & & \\
\hline $\mathbf{1 9 9 2}$ & & & & & & & & & \\
\hline $\mathbf{1 9 9 3}$ & & & & & & & & & \\
\hline $\mathbf{1 9 9 4}$ & & & & & & & & & \\
\hline $\mathbf{1 9 9 5}$ & & & & & & & & & \\
\hline $\mathbf{1 9 9 6}$ & $3,461.073$ & $3,454.714$ & $3,449.825$ & $3,437.633$ & $3,423.234$ & $3,404.152$ & & & \\
\hline $\mathbf{1 9 9 7}$ & $3,461.082$ & $3,454.720$ & $3,449.829$ & $3,437.642$ & $3,423.238$ & $3,404.162$ & & & \\
\hline $\mathbf{1 9 9 8}$ & $3,461.091$ & $3,454.730$ & $3,449.835$ & $3,437.648$ & $3,423.242$ & $3,404.173$ & & & \\
\hline $\mathbf{1 9 9 9}$ & $3,461.105$ & $3,454.744$ & $3,449.848$ & $3,437.657$ & $3,423.247$ & $3,404.169$ & & & \\
\hline $\mathbf{2 0 0 0}$ & $3,461.109$ & $3,454.749$ & $3,449.853$ & $3,437.660$ & $3,423.250$ & $3,404.157$ & & & \\
\hline $\mathbf{2 0 0 1}$ & $3,461.111$ & $3,454.752$ & $3,449.856$ & $3,437.663$ & $3,423.254$ & $3,404.152$ & & & \\
\hline $\mathbf{2 0 0 2}$ & $3,461.113$ & $3,454.754$ & $3,449.857$ & $3,437.665$ & $3,423.256$ & $3,404.150$ & & & \\
\hline $\mathbf{2 0 0 3}$ & $3,461.112$ & $3,454.752$ & $3,449.856$ & $3,437.665$ & $3,423.256$ & $3,404.137$ & & & \\
\hline $\mathbf{2 0 0 4}$ & $3,461.117$ & $3,454.754$ & $3,449.858$ & $3,437.668$ & $3,423.257$ & $3,404.127$ & & & \\
\hline
\end{tabular}

* The 1986 elevations that appear in all reports prior to the 2001 report are from a report filed by Jerry Williams (3/89), Geoscience Dept. Those elevations were, in turn, taken from the 1987 data, rounded to two decimal places and referenced as 1986 . When this was discovered it was decided to remove the 1986 information from all subsequent reports. 